\title{
Severe Nocturnal Cardiac Arrhythmias Caused by Sleep Apnea- INDUCED HYPOXEMIA IN MALES
}

\author{
E. Szaboova ${ }^{1}$, Z. Tomori², P. Szabo ${ }^{3}$, S. Gresova ${ }^{2}$, V. Donic ${ }^{2}$ \\ ${ }^{1}$ Clinic of Internal Medicine, Faculty of Medicine and ${ }^{2}$ Department of Physiology and Sleep Laboratory, Faculty of Medicine, Safarik \\ University; ${ }^{3}$ Faculty of Aeronautics, Technical University, Kosice, Slovakia
}

\begin{abstract}
Nocturnal cardiac arrhythmias (NCA) were analyzed in patients with sleep apnea/hypopnea syndrome (SAHS) and controls. Occurrence and severity of NCA were compared in 33 SAHS patients and 16 control subjects, matched for cardiovascular risk factors. Continuous overnight polysomnography provided ECG, respiratory and sleep parameters for a comparative analysis. Various types and severity of NCA were detected already in moderate SAHS (apnea/hypopnea index $=26 \pm 15.6 / \mathrm{h}$ ), reflecting the respiratory and atherosclerotic changes. Moderately severe arrhythmias, represented with benign and 2 complex types were caused by hypoxemia characterized by AHI, minimal $\mathrm{SaO}_{2}$, and lower values after desaturation. Three-time higher prevalence of complex arrhythmias in SAHS patients was not significantly different by usual statistical comparison, likely due to a low number of controls and a joint occurrence of various types and complex severity of arrhythmias in some patients. Therefore, a complex assessment of different types and varying severity of arrhythmias would require a scale specifically constructed for their evaluation.
\end{abstract}

Key words: cardiac arrhythmias sleep apnea, hypoxemia, polysomnography, carotid ultrasonography

\section{INTRODUCTION}

Several epidemiological studies have indicated a connection between the sleep apnea/hypopnea syndrome (SAHS) and cardiovascular (CV) diseases, resulting in increased morbidity and mortality. Nocturnal cardiac arrhythmias (NCA) are frequent and represent a potential risk in cases of severe sleep apnea, particularly during long obstructive apneic events. There is increased risk for the development of various malignant arrhythmias, when sleep apnea is associated with preexisting structural cardiac changes [1]. There are conflicting data concerning the prevalence and severity of NCA in patients with SAHS. While some authors have detected an increase in both brady- and tachyarrhythmias, others have found their low incidence in patients without severe cardiac or pulmonary diseases. No clear epidemiological evidence for a causal relation between SAHS and arrhythmias has been found when confounders were considered [2]. Nevertheless, most experts agree that cardiac arrhythmias occur more frequently in patients with SAHS and that their incidence increases with the degree of arterial oxygen desaturation and the number of apnoeic episodes [3]. General mechanisms and cardio-respiratory as well as neurophysiological and sleep parameters contributing to development of arrhythmias are illustrated in Fig 1.

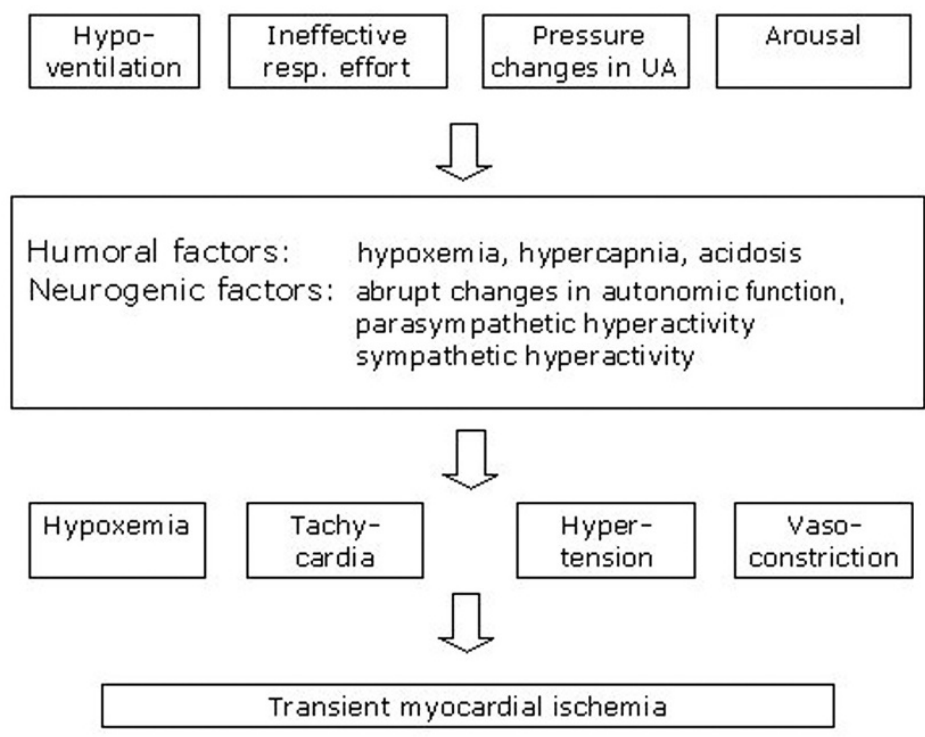

Fig. 1. General mechanisms and cardio-respiratory and neuro-physiological effects of sleep disordered breathing. 
The aim of the present study was to assess and compare the occurrence and severity of NCA in subjects with and without moderate SAHS, matched for traditional risk factors for atherosclerosis (AS) and CV pathology. There are many studies analyzing separately the occurrence and severity of cardiac arrhythmias in patients with sleep apnea, but there is no method for a complex assessment and a quantitative comparison of different types and severity of arrhythmias in patients with SAHS.

\section{Material AND Methods}

The study was performed in accordance with the Declaration of Helsinki for Human Research. The protocol obtained approval of the Ethics Committee of Medical Faculty Safarik University in Kosice, Slovakia and informed consent was obtained from all the subjects enrolled into the study.

\section{ANTHROPOMETRIC, LABOratory, AND CLINICO-}

Physiological Characteristics of Subjects

Adult males were investigated in this study, because of the lower prevalence of SAHS in middle-aged females. Thirty-three males with moderate SAHS and 16 subjects without SAHS, matched for specific CV co-morbidity (apparently healthy adults and patients with arterial hypertension - AH or coronary artery diseases CAD) were studied. Overnight polysomnography including simultaneous electrocardiogram (ECG) was performed in the sleep laboratory of P.J. Safarik University in Kosice, Slovakia. The subjects underwent careful laboratory and clinical examination, including analysis of fasting venous blood samples, after their informed consent in cardiologic outpatient department of the $4^{\text {th }}$ Clinic of Internal Medicine the day after polysomnographic recording. The medical history, physical examination and clinical documentation of subjects were evaluated. Current and past dietetic and pharmacological treatments were also recorded. Exclusion criteria included insufficiency of vital organs, severe bronchial asthma, complicated hematological disorders, endocrinopathy, diabetes mellitus (DM) type 1 or 2 with specific chronic complications or treated with insulin, malignant diseases, marked neurological abnormalities, current use of antiarrhythmic drugs, and non-compliance. None of the patients were alcoholic, nor were treated with nasal continuous positive airway pressure (nCPAP).

Traditional risk factors for AS (age, positive family history of premature CV diseases, hyperlipoproteinaemia - HLP, DM, AH, smoking habit, and obesity, were defined according to international guidelines [4]. Height, weight, waist-to-hip ratio (WHR), and body mass index $\left(\mathrm{BMI}=\mathrm{kg} / \mathrm{m}^{2}\right)$ were measured following a standardized protocol [5]. CAD was based on the presence of clinical symptoms, positive exercise ECG, thallium scan, and/or the finding of significant $(>50 \%)$ stenosis on coronarography [6].

Intimo-media thickness (IMT) of common carotid arteries as a marker of early AS has strong association with major CV risk factors. For its high specificity and negative predictive value, determination of IMT pro- vides a useful screening test in population with low prevalence of CAD at a cut-off point $\geq 0.85 \mathrm{~mm}$ for the prediction of severe CAD in symptomatic patients. A real-time triplex, high-resolution B-mode ultrasonography (AU4 Idea, Esaote Biomedica, 7.5 MHz probe) was used. The IMT was measured from magnified $(5 x)$ images at the maximum thickness of the far arterial wall during the end-diastole. A plaque was defined as a localized lesion of the artery (echogenic structure encroaching into the vessel lumen) with IMT thickness $>1.2 \mathrm{~mm}$. The largest value $\left(\mathrm{IMT}_{\max }\right)$ out of 4 measurements as well as the occurrence of IMT $\geq 0.85 \mathrm{~mm}$ were used to detect the earliest signs of AS. Finally, all extra-cranial vessels were screened for presence of stenotic lesions (advanced AS). More details are described in a previous paper [7].

\section{SLEEP STUdies}

Overnight polysomnographic recording was performed in a sleep laboratory with Alice 3 device (Respironics, Pittsburgh, PA) using a standard methodology. More than 20 parameters were continuously recorded in parallel. Indices calculated per hour of sleep were as follows: apnea-hypopnea index (AHI), indicating the number of apnoeic and hypopneic episodes, arousal index (ArI) expressing the number of arousals lasting 3-15 s. Other parameters were: minimal and average nocturnal oxygen saturation $\left(\mathrm{MinSaO}_{2}\right.$, AvgSaO $\mathrm{S}_{2}$, mean oxygen saturation after desaturations by $>3 \%\left(\mathrm{DeSaO}_{2}\right)$, and the time in minutes spent with $\mathrm{SaO}_{2}$ below 90\%. Micro-arousals and macro-arousals lasting $<3 \mathrm{~s}$ and $>15 \mathrm{~s}$, respectively, were also identified [8]. The recorded parameters were stored on optical disc and automatically analyzed by software and corrected by experts after visual analysis to eliminate occasional artifacts. Respiratory events were defined from polysomnographic records as a decrease in airflow to amplitude $<20 \%$ (apnea) or $<70 \%$ (hypopnea) of the baseline lasting $>10 \mathrm{~s}$ and associated with oxygen desaturation by $>3 \%$. Apneic episodes were identified if airflow was absent for $>10 \mathrm{~s}$ and the presence of $>10$ apneas or hypopneas/hour of sleep is characteristic for SAHS syndrome [8]. Healthy persons present apneahypopnea episodes occasionally $(<5 / \mathrm{h})$. More details are given in a previous publication [7].

\section{ECG ANALYSIS}

ECG records from a complete overnight polysomnogram monitored continuously before and during sleep were analyzed by a cardiologist in a blinded fashion to the clinical data and classification of SAHS. We assessed the occurrence, type, and frequency of arrhythmias in each subject and compared the results obtained in SAHS patients with the values of healthy controls and patients without SAHS, matched for various CV risk factors and diseases. Each arrhythmia was determined in relation to the apnea, sleep stage, oxyhemoglobin saturation, as well as minimum and maximum heart rate and $\mathrm{R}-\mathrm{R}$ interval (during a $\approx 30 \mathrm{~s}$ period before and after the onset of arrhythmia). Apnearelated arrhythmia was defined, when the arrhythmia occurred at the onset, during, or till $10 \mathrm{~s}$ after termina- 
tion of apnea. Non-apnea-related arrhythmias have been evaluated separately. The results of a complex estimation of 3 different types and various degrees of severity of NCA have been described elsewhere [9].

\section{Statistical Methods}

The results were expressed as means $\pm \mathrm{SD}$ and analyzed by usual statistical methods from a package STATISTICA 1999. The significance of the mean differences in characteristics between the SAHS and nonSAHS subjects was determined by using the nonparametric Mann Whitney U test for continuous parameters. Chi-squared test was used for an assessment of proportions. If the number of a variable was less than 5 , the Fisher exact test was used. The difference was considered as significant at $\mathrm{P}<0.05$.

\section{RESULTS}

Our patients with moderate SAHS did not differ from subjects without SAHS in various CV risk factors, diseases, and their therapy (Table 1). The mean values of polysomnographic parameters demonstrated a much higher AHI $(\mathrm{P}<0.0001)$ by definition in SAHS patients, compared with practically normal nocturnal breathing in the control group, including subjects with some CV pathology (Table 2). SAHS patients had more severe nocturnal hypoxemia with lower $\mathrm{MinSaO}_{2}$ $(\mathrm{P}<0.0005)$, and $\mathrm{DeSaO}_{2}(\mathrm{P}<0.05)$, and a tendency to

Table1. Baseline, anthropometric, laboratory, and clinical characteristics of subjects.

\begin{tabular}{|c|c|c|c|}
\hline & $\begin{array}{l}\text { SAH (+) } \\
\mathrm{n}=33\end{array}$ & $\begin{array}{l}\text { SAHS (-) } \\
\mathrm{n}=16\end{array}$ & $\mathbf{P}$ \\
\hline Age (yr) & $52 \pm 8$ & $48 \pm 11$ & NS \\
\hline BMI $\left(\mathrm{kg} / \mathrm{m}^{2}\right)$ & $28.9 \pm 4.4$ & $29.7 \pm 4.4$ & NS \\
\hline Waist circumference (cm) & $102 \pm 10$ & $100 \pm 12$ & NS \\
\hline Total cholesterol (mmol/l) & $5.5 \pm 1.01$ & $5.1 \pm 0.8$ & NS \\
\hline TriAcylGlyc (mmol/l) & $2.6 \pm 3.9$ & $1.9 \pm 0.9$ & NS \\
\hline HDL-chol (mmol/l) & $1.2 \pm 0.3$ & $1.2 \pm 0.3$ & NS \\
\hline LDL-chol (mmol/l) & $3.7 \pm 1.2$ & $3.1 \pm 0.7$ & NS \\
\hline Glycemia (mmol/l) & $5.6 \pm 0.8$ & $5.6 \pm 1.2$ & NS \\
\hline Age risk ( $\geq 45$ years) (n/\%) & $28 / 85$ & $9 / 56$ & NS \\
\hline Positive family history (n/\%) & $7 / 21$ & $2 / 13$ & NS \\
\hline $\mathrm{AH}(\mathrm{n} / \%)$ & $21 / 64$ & $7 / 44$ & NS \\
\hline $\mathrm{DM}(\mathrm{n} / \%)$ & $2 / 6$ & $3 / 19$ & NS \\
\hline $\operatorname{HLP}(\mathrm{n} / \%)$ & $18 / 55$ & $9 / 56$ & NS \\
\hline Obesity (BMI) (n/\%) & $9 / 27$ & $6 / 38$ & NS \\
\hline Central obesity (Waist) (n/\%) & $14 / 42$ & $7 / 44$ & NS \\
\hline Smoking $(\mathrm{n} / \%)$ & $19 / 58$ & $10 / 63$ & NS \\
\hline ACE inhibitors (n/\%) & $11 / 33$ & $5 / 31$ & NS \\
\hline Beta-blockers (n/\%) & $11 / 33$ & $9 / 56$ & NS \\
\hline Ca-channel blockers (n/\%) & $10 / 30$ & $4 / 25$ & NS \\
\hline
\end{tabular}

BMI - body mass index, RF-AS - risk factors for atherosclerosis, AH - arterial hypertension, DM - diabetes mellitus, HLP hyperlipoproteinemia, use of antihypertensive drugs in subjects with and without SAHS, matched for risk factors. longer time spent with $\mathrm{SaO}_{2}<90 \%(\mathrm{P}=0.14)$ and to lower values of $\mathrm{AvgSaO}_{2}(\mathrm{P}=0.505)$.

Such intermittent hypoxemic episodes caused by apnea-hypopnea events resulted in more severe NCA already in moderate SAHS patients (AHI $26 \pm 15.6 / \mathrm{h}$ ) than in non-SAHS subjects. With regard to the character of arrhythmias, benign types predominated and no malignant arrhythmias were found in our subjects. The most severe types of arrhythmias had complex forms as follows: 1) supraventricular (SV) tachyarrhythmia: a short run of SV tachycardia in one of the 8 patients in a subgroup with SAHS and CAD;2) ventricular tachyarrhythmia: bigeminy in 2 of the 14 patients with SAHS and AH, 3 of the 8 in a subgroup with SAHS and CAD, and only in one person from the non-SAHS group, who had CAD. The occurrence of complex arrhythmias was 6 of the 33 $(18.2 \%)$ in SAHS patients vs. 1 of the $16(6.25 \%)$ in non-SAHS group $(\mathrm{P}>0.05)$. A nearly 3 times higher prevalence of complex forms of arrhythmias was detected in our SAHS patients, compared with nonSAHS subjects.

Table 2. Carotid ultrasonographic data and cardiovascular diseases as well as respiratory and sleep parameters in subjects with and without SAHS.

\begin{tabular}{|c|c|c|c|}
\hline & SAHS (+) & SAHS (-) & $\mathbf{P}$ \\
\hline $\mathrm{IMT}_{\max }(\mathrm{mm})$ & $0.91 \pm 0.21$ & $0.77 \pm 0.18$ & $<0.05$ \\
\hline $\mathrm{IMT}>0.85 \mathrm{~mm}(\mathrm{n} / \%)$ & $18 / 54.5$ & $4 / 25$ & $<0.05$ \\
\hline Sten $1(\mathrm{n} / \%)$ & $10 / 30$ & $4 / 25$ & NS \\
\hline Sten $2(\mathrm{n} / \%)$ & $2 / 6$ & $0 / 0$ & NS \\
\hline $\mathrm{CAD}(\mathrm{n} / \%)$ & $8 / 24$ & $5 / 31$ & NS \\
\hline Previous MI (n/\%) & $5 / 15$ & $3 / 19$ & NS \\
\hline $\mathrm{AHI}(\mathrm{n} / \mathrm{h})$ & $26.0 \pm 15.6$ & $4.1 \pm 2.6$ & $<0.001$ \\
\hline Arousal index $(\mathrm{n} / \mathrm{h})$ & $22.2 \pm 13.7$ & $14.9 \pm 13.3$ & NS \\
\hline $\mathrm{MinSaO}_{2}(\%)$ & $76.7 \pm 10.5$ & $87.0 \pm 6.5$ & $<0.001$ \\
\hline $\mathrm{AvgO}_{2}(\%)$ & $90.5 \pm 4.8$ & $91.8 \pm 2.4$ & NS \\
\hline $\mathrm{DeSaO}_{2}(\%)$ & $85.7 \pm 4.9$ & $88.4 \pm 4.8$ & $<0.05$ \\
\hline Total sleep time (h) & $5.4 \pm 1.98$ & $5.7 \pm 2.0$ & NS \\
\hline $\mathrm{SaO}_{2}<90 \%(\%)$ & $18.8 \pm 19.5$ & $14.9 \pm 13.7$ & NS \\
\hline
\end{tabular}

IMT - intimomedia thickness, Sten - stenosis $1=$ up to $40 \%$, Sten - stenosis $2=40-60 \%$, CAD - coronary artery disease, $\mathrm{MI}$ - myocardial infarction, $\mathrm{SaO}_{2}$ - arterial oxygen saturation.

\section{Discussion}

\section{Previous Data on ArRhythmogenesis in SAHS}

The present study provides insights into the complicated relationships between SAHS and development of various NCA, which are common [10], but generally benign [11]. Bradycardic forms, such as sinus bradycardia, sinus pauses up to $2 \mathrm{~s}$ in duration, and second degree atrio-ventricular block (AVB) type 1 are commonly observed during normal sleep, and are deemed benign [12]. However, patients with SAHS had more nocturnal and diurnal ST-segment depression as well as sinus bradycardia, sinus tachycardia, premature supra-ventricular complexes (PSVC), supra-ventricular 
tachycardia, and couplets than the snoring and control groups [11].

Most experts agree that arrhythmias occur more frequently in patients who have sleep apnea and that the incidence increases with the number of apneic episodes and the degree of arterial oxygen desaturation [13]. In $88 \%$ of patients referred for pacemaker therapy with asymptomatic bradyarrhythmias occurring during sleep, apnea episodes were documented by overnight polysomnography [14]. Mild SAHS was detected in 26\% and moderate to severe SAHS in 34\% of patients with ventricular arrhythmias, but without heart failure [15].

Most severe arrhythmias, such as persistent SV tachycardia, atrial fibrillation (AF) or flutter, and paroxysmal ventricular tachycardia seem more likely to occur in the setting of pre-existing structural heart disease [10]. Sleep disordered breathing was found in approximately half of patients with AF, which prevalence is substantially greater than the occurrence of SAHS in patients with established CV disease but without past or current AF [16].

Some studies have demonstrated objective benefits from CPAP therapy on cardiac rhythm disturbances, especially bradyarrhythmic forms [14, 17-20]. Even serious and potentially fatal arrhythmias occurring during sleep in patients with SAHS were attenuated by effective treatment $[18,20]$. Electrophysiological studies detected no sustained or structural conduction system abnormalities in most of patients with significant bradycardia caused by SAHS, but impaired initiation of impulses by the sinus node [18]. When treated with CPAP, these patients have an excellent prognosis with regard to five year risk of syncope or cardiac arrest, strongly supporting a causal relationship between SAHS and at least this form of cardiovascular morbidity [18]. We documented more frequent, more severe, and longer duration of hypoxia in our SAHS patients with more severe arrhythmias.

Most of cardiac rhythm disturbances in patients with SAHS showed a significant correlation with nocturnal minimum $\mathrm{SaO}_{2}$, sleep fragmentation, and urinary catecholamine excretion. Moreover ST-segment depressions were related to sleep fragmentation and the sympathetic tone [11]. Accordingly, cardiac arrhythmias were related to the level of sleep-disordered breathing and oxygen desaturation in a morbidly obese population [21], in patients with severe SAHS (AHI $>30$ ), prolonged periods of arterial oxyhaemoglobin desaturation, and in patients with low diurnal awake $\mathrm{PaO}_{2}$ [22], and particularly during REM sleep [19]. An analysis of factors involved in the pathogenesis of heart blocks in patients with sleep apnea indicated that almost $90 \%$ of these episodes occur during REM sleep and during an oxygen desaturation of at least 4\% [17].

\section{ANALysis of OUR Results}

Our study documented a low prevalence of complex forms of arrhythmia in moderate sleep apneics (AHI $<30 / \mathrm{h}$ ) with normal left ventricular (LV) function. The prevalence of rhythm disturbances in SAHS patients was found not significantly different from that in non-apneic snorers or in the general population [2]. Such discrepant results can be explained in terms of selection bias. Moreover, the different CV co-morbidity of study population and the methodology used for detection of SAHS, as well as discrepancy in the assessment of the severity of both SAHS and arrhythmias further enhance the difficulty to compare various studies [2]. However, a significant difference between the groups with and without SAHS, matched for various risk factors, could not be proved by a separate analysis of 3 different types and various severities of NCA induced by intermittent hypoxemia using simple statistical comparison. This can be explained by a low prevalence, a moderate severity of arrhythmias, a small number of subjects, various structural and functional $\mathrm{CV}$ changes, but mainly by a joint occurrence of various types and severity of arrhythmias in some subjects. Therefore, a complex estimation of varying severity of NCA using a special 7-grade scale was tested and various structural and functional CV changes have been analyzed in a parallel publication [9]. Fig. 2 illustrates the great importance of REM sleep and the accompanying apneic episodes and hypoxemia for the induction of NCA in one of our patients.

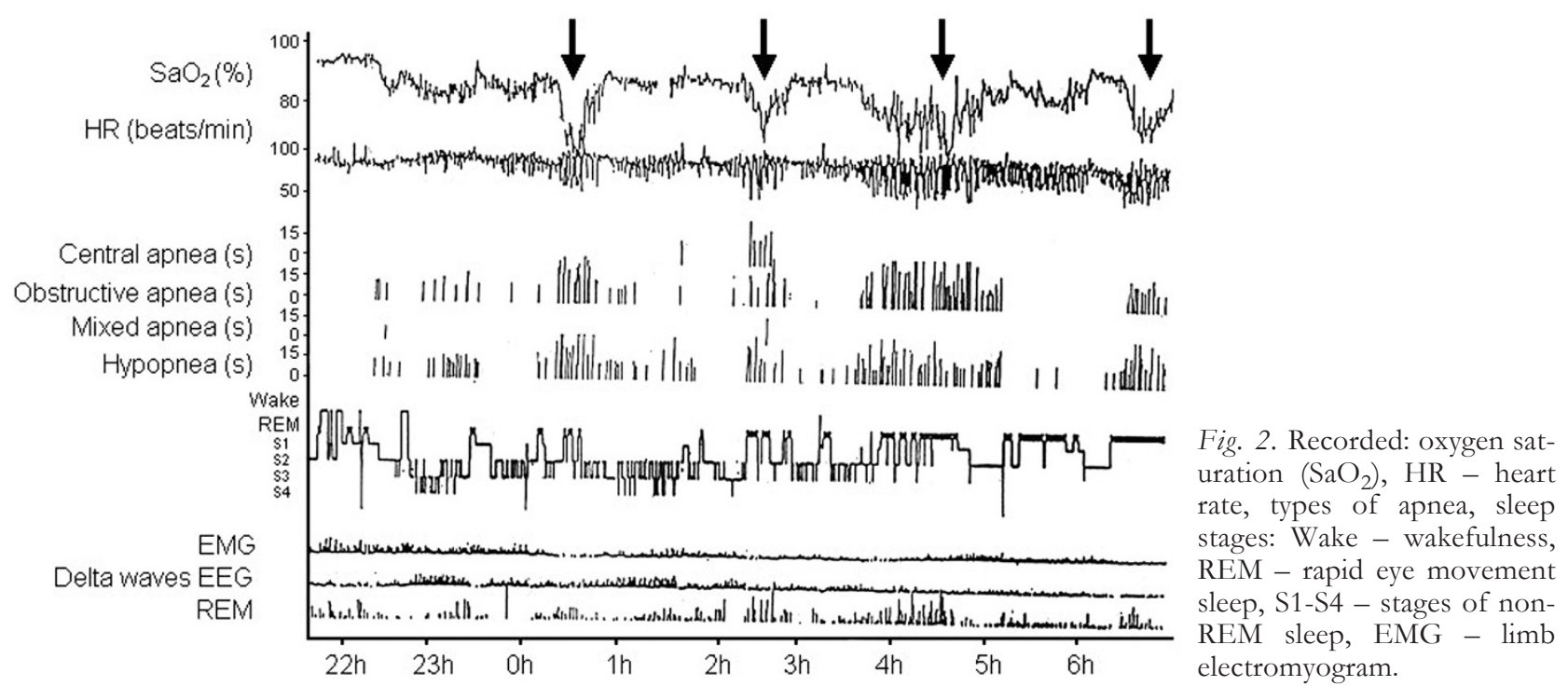




\section{SUMMARY}

Moderate SAHS in our patients proved to generate severe NCA by modifying the heart function and/or even the structure, which was a result of an action of potent triggering mechanisms. However, more material collected from strictly selected subjects in a prospective study with the evaluation of a large number of confounding factors is needed for a complex analysis of the causal relationship between SAHS and the severity of NCA. Such a complex analysis requires the use of a method with a multi-grade scale for a quantitative assessment of various types and different degrees of the severity of NCA.

Acknowledgements: The study was supported by grants APVV 20-047705 and COST Action B-26.

Conflicts of interest: The authors had no conflict of interest to declare in relation to this article.

\section{REFERENCES}

1. Hermann JP. Sleep apnea and cardiovascular diseases. In: Guilleminault C, Partinen M (eds.): Obstructive Sleep Apnea Syndrome. New York, Raven Press, 1990; 81-98.

2. Flemons WW, Remmers JE, Gillis AM. Sleep apnea and cardiac arrhythmias: is there a relationship? Am Rev Respir Dis 1993; 148: 618-21.

3. Hoffstein V, Mateika S. Cardiac arrhythmias, snoring, and sleep apnea. Chest 1994; 106: 466-71.

4. Guidelines for the management of arterial hypertension. Eur Heart J 2007; 28: 1462-536.

5. Babcak M, Merciakova M, Kmec M, Jenca J. Type 2 diabetes mellitus and arterial hypertension - recent findings. Diabetes a obezita 2004; 4: 15-41 (Article in Slovak).

6. Schulz R, Seeger W, Fegbeutel C, Husken H, Bodeker $\mathrm{RH}$, Tillmanns H, Grebe M. Changes in extracranial arteries in obstructive sleep apnoea. Eur Resp J 2005; 25: 69-74.

7. Szaboova E, Tomori Z, Donic V, Petrovicova J, Szabo P. Sleep apnoea inducing hypoxemia is associated with early signs of carotid atherosclerosis in males. Respir Physiol Neurobiol 2007; 155: 121-27.

8. American Sleep Disorders Association (ASDA) Report. Sleep-related breathing disorders in adults: recommendations for syndrome definition and measurement techniques in clinical research. Sleep 1999; 22: 667-89.

9. Szaboova E, Tomori Z, Donic V, Gresova S, Szabo P. Complex estimation of various types and severity of nocturnal cardiac arrhythmias caused by moderate sleep apnoea. Eur Respir J 2009; 34 Suppl 53: 652s (Abstract).

10. Quan SF, Gersh BJ. Cardiovascular consequences of sleep-disordered breathing: Past, present and future: Report of a workshop from the national center on sleep disorders research and the national heart, lung, and blood institute. Circulation 2004; 109: 951-7.

11. Alonso-Fernandez A, Garcia-Rio F, Racionero MA, Pino JM, Ortuno F, Martinez I, Villamor J. Cardiac rhythm disturbances and ST-segment depression episodes in patients with obstructive sleep apnea-hypopnea syndrome and its mechanisms. Chest 2005; 127; 15-22.
12. Gula LJ, Krahn AD, Skanes AC, Yee R, Klein GJ. Clinical relevance of arrhythmias during sleep: guidance for clinicians. Heart 2004; 90: 347-52.

13. Cutler MJ, Hamdan AL, Hamdan MH, Ramaswamy K, Smith ML. Sleep apnea: from the nose to the heart. J Am Board Fam Pract 2002; 15:128-41.

14. Stegman SS, Burroughs JM, Henthorn RW. Asymptomatic bradyarrhythmias as a marker for sleep apnea: appropriate recognition and treatment may reduce the need for pacemaker therapy. Pacing Clin Electrophysiol 1996; 19: 899-904.

15. Koshino Y, Satoh M, Katayose Y, Yasuda K, Tanigawa T, Takeyasu N, Watanabe S, Yamaguchi I, Aonuma K. Association of sleep-disordered breathing and ventricular arrhythmias in patients without heart failure. Am J Cardiol 2008; 101: 882-6.

16. Gami AS, Pressman G, Caples SM, Kanagala R, Gard JJ, Davison DE, Malouf JF, Ammash NM, Friedman PA, Somers VK. Association of atrial fibrillation and obstructive sleep apnea. Circulation 2004; 110: 364-7.

17. Koehler U, Fus E, Grimm W, Pankow W, Schäfer H, Stamnitz H, Peter JH. Heart block in patients with obstructive sleep apnoea: Pathogenetic factors and effects of treatment. Eur Respir J 1998; 11: 434-9.

18. Harbison J, O'Reilly P, McNicholas WT. Cardiac rhythm disturbances in the obstructive sleep apnea syndrome: ef fects of nasal continuous positive airway pressure therapy. Chest 2000; 118: 591-5.

19. Kanagala R, Murali NS, Friedman PA, Ammash NM, Gersh BJ, Ballman KV, Shamsuzzaman AS, Somers VK. Obstructive sleep apnea and the recurrence of atrial fibrillation. Circulation 2003; 107: 2589-94.

20. Kohler M, Pepperell JCT, Casadei B, Craig S, Crosthwaite N, Stradling JR, Davies JRO. CPAP and measures of cardiovascular risk in males with OSAS. Eur Respir J 2008; 32: 1488-96.

21. Valencia-Flores M, Orea A, Castano VA, Resendiz M, Rosales M, Rebollar V. Prevalence of sleep apnea and electrocardiographic disturbances in morbidly obese patients. Obes Res 2000; 8: 262-9.

22. Roche F, Xoung AN, Court-Fortune I, Costes F, Pichot V, Duverney D, Vergnon JM, Gaspoz JM, Barthelemy JC. Relationship among the severity of sleep apnea syndrome, cardiac arrhythmias, and autonomic imbalance. Pacing Clin Electrophysiol 2003; 26: 669-77.

Address for correspondence:

Zoltan Tomori

Department of Physiology

Faculty of Medicine

UPJS, Tr. SNP 1

04066 Kosice

Slovakia

Phone/fax: +4216423763

E-mail: zoltan.tomori@gmail.com 